\section{PWE-067 WATER INSUFFLATION VS NARROW BAND IMAGING, WHICH MODALITY PRODUCES RELIABLE IMAGES OF TERMINAL ILEUM AT COLONOSCOPY?}

doi:10.1136/gutjnl-2013-304907.356

1." $\mathrm{M}$ H Thoufeeq, ${ }^{1} \mathrm{~N}$ Kumar. 'Endoscopy/Gastroenterology, Peterborough and Stamford Hospitals NHS Foundation Trust, Peterborough, UK

Introduction Terminal ileal (TI) images are reliable indicators of completeness of colonoscopy ${ }^{1}$. Studies have shown that TI image provide more convincing than caecal images to verify completion of colonoscopy ${ }^{2}$. Acquisition of TI images after water insufflation (WI) has been found to produce reliable images ${ }^{3}$. We wanted to identify if TI images after WI through the biopsy channel or narrow band images of TI produce more reliable as part of image documentation. Methods 4 images of terminal ileum were captured using Olympus colonoscope $($ B. They were 1 captured with conventional white light endoscopy (WLE), 1 captured with white light endoscopy + water insufflation (WLEWI), 1 captured with narrow band imaging (NBI) and 1 captured with NBI + water insufflation (NBIWI).

Without informing that these were TI images, the 4 terminal images were sent to endoscopists by email along with an invitation to participate in a survey using survey monkey ${ }^{\circledR}$ portal. Using the survey the respondents marked each image as follows: not terminal ileum $=1$, may be terminal ileum $=2$, most likely terminal ileum $=3$, definitely terminal ileum $=4$.

Results 36 endoscopists completed our survey. 49\% were gastroenterology Specialist registrar, 40\% were consultant gastroenterologists and $11 \%$ were colorectal surgeons. Most of the respondents $(\mathrm{n}=31)$ were from the UK, 4 were from the United States and 1 was from the rest of the Europe.

$19.4 \%(n=7)$ of respondents had colonoscopy experience of $1-2$ years, $30.6 \%(n=11)$ had colonoscopy experience of $3-5$ years, $19.4 \%(n=7)$ had colonoscopy experience of $5-9$ years $30.6 \%$ $(\mathrm{n}=11)$ had colonoscopy experience of more than 10 years.

Responses in Table 1:

\section{Abstract PWE-067 Table 1}

\begin{tabular}{lllll}
\hline & WLE & WLEWI & NBI & NBIWI \\
\hline Not TI (Score 1) & $5.6 \%$ & $0.0 \%$ & $2.8 \%$ & $2.8 \%$ \\
May be TI (score 2) & $33.3 \%$ & $16.7 \%$ & $22.2 \%$ & $8.3 \%$ \\
Most likely TI (score 3) & $38.9 \%$ & $22.2 \%$ & $22.2 \%$ & $22.2 \%$ \\
Definitely TI (score 4) & $22.2 \%$ & $61.1 \%$ & $52.8 \%$ & $66.7 \%$ \\
\hline
\end{tabular}

Conclusion Water insufflation on terminal ileum using the biopsy channel of colonoscope produce more reliable images compared to White light or narrow band images (NBI). Combination of NBI and water insufflation together produced the most reliable images.

Disclosure of Interest None Declared.

\section{REFERENCES}

1. Cherian $S$, Singh P. Is routine ileoscopy useful? An observational study of procedure times, diagnostic yield, and learning curve. Am J Gastroenterol 2004; 99(12):23242329.

2. Powell N, Knight H, Dunn J, Saxena V, Mawdsley J, Murray C et al. Images of the terminal ileum are more convincing than cecal images for verifying the extent of colonoscopy. Endoscopy 2011; 43(3):196-201.

3. Baraza W, Brown S, Shorthouse AJ, Tiffin N, Hurlstone DP. Direct photographic documentation of ileal mucosa in routine colonoscopy is not an independent valid or reliable proof of completion: quality assurance issues for the national colorectal cancer-screening programme. Colorectal Dis 2009; 11(1):89-93.

\section{PWE-068 DIAGNOSTIC YIELD OF SERIOUS DISEASE AND VARIATION IN ELECTIVE GASTROSCOPY RATES IN ENGLISH GENERAL PRACTICE: ANALYSIS OF ADMINISTRATIVE DATA}

doi:10.1136/gutjnl-2013-304907.357
." M Shawihdi, ${ }^{2} \mathrm{E}$ Thompson, ${ }^{3} \mathrm{~N}$ Kapoor, ${ }^{2} \mathrm{G}$ Powell, ${ }^{2} \mathrm{M}$ Roughton, ${ }^{3} \mathrm{R}$ Sturgess, ${ }^{3} \mathrm{~N}$ Stern, ${ }^{2} \mathrm{M}$ Pearson, ${ }^{1} \mathrm{~K}$ Bodger. 'Gastroenterology Department, University of Liverpool; ' 2 Aintree Health Outcomes Partnership; ${ }^{3}$ Digestive Diseases Centre, Aintree University Hospital, Liverpool, UK

Introduction The optimum role for gastroscopy (OGD) in managing dyspepsia and detecting oesophagogastric cancer (OG-Ca) is controversial. UK general practitioners (GPs) serve a gatekeeper role in selecting dyspeptic patients for OGD. We reported that variation in rates of OGD at the level of GP practise populations is associated with OG-Ca outcome, specifically that low rates are related to risk of poor outcome.[1] We wished to show that GP practises with low OGD rates are likely to be operating more selective referral practise with higher yield of serious pathology.

Methods GP practises with $\geq 1$ incident case of OG-Ca were selected, as described.[1.2] Using a two-year download of HES data we identified all elective OGD procedures and obtained practise data to calculate age-sex adjusted OGD rates. Practices were divided into OGD rate tertiles (Low, Medium or High). An algorithm was developed to analyse coded diagnoses for first OGDs, identifying most "serious" condition: (1) OG-Ca, (2) Major acid-peptic diseases, (3) Minor findings (e.g. gastritis), (4) Benign GI neoplasms, (5) Upper GI symptom codes, (6) Miscellaneous (all others). We compared age and proportions with serious disease (categories: 1-2) across the GP practise tertiles.

Results 587,256 patients had elective OGD from 6,513 practises serving an adult population of c.39 million. Overall, yield of OG-Ca was $2.1 \%$, major acid-peptic diseases $11.6 \%$ and the remaining $86.3 \%$ were mainly minor pathologies or symptom codes. Mean OGD rate for Low, Middle, High practises: 4.4 vs 8.1 vs 12.9 per 1,000 population. No difference in age distribution of populations across tertiles. Mean age of patients undergoing OGD was highest for low tertile practises (60.2 vs 59.5 vs 58.4 yrs; $p<0.001)$ which had highest yield of serious disease: $16,595 / 108,679(15.3 \%)$ vs $28,177 / 203,771$ (13.9\%) vs 36,026/274,806 (13.1\%) ( $<<0.001)$.

Conclusion Low referring practises appear to target slightly older patients and achieve higher yield of serious disease. Although higher yield may be more consistent with current guidelines, it may also indicate an increased risk of referral at a later stage in the disease process and of poorer OG-Ca outcome.[1]

Disclosure of Interest None Declared.

\section{REFERENCES}

1. Shawihdi, M., et al. Gastroscopy rate in English general practise populations: association with outcome for oesophagogastric cancer. Gut, 2012. 61 (Suppl 2):A19.

2. Shawihdi, M., et al. Emergency Hospital Admission as a Route for Oesophagogastric Cancer Diagnosis: A Marker of Poor Outcome and a Candidate Quality Indicator for Local Services. Gastroenterology, 2011. 140( 5, Supplement 1):S207.

\section{PWE-069 FACTORS ASSOCIATED WITH REPORTED PAIN DURING COLONOSCOPY: A RETROSPECTIVE STUDY}

doi:10.1136/gutjnl-2013-304907.358

1."N Rahman, ' 1 A Cuison, 'L satkunananthan, ${ }^{20}$ Shabir, 'A Zaman, 'S varma, 'M A Zubir. 'Conquest Hospital, St Leonards on sea, UK; ${ }^{2}$ General Surgery, Conquest Hospital, St Leonards on sea, UK

Introduction Colonoscopy is generally perceived as being a painful procedure. Nonetheless, pain is a rather subjective experience for individuals and can be multifactorial. The aim of this study was to identify the predictors of pain during colonoscopy.

Methods 1824 consecutive colonoscopies performed in 2010 were identified. Data was retrospectively collected on gender, patients medical co-morbidities, type and effectiveness of bowel preparation, medication used during the procedure, endoscopist's performance and endoscopic findings such as presence of diverticular disease. Logistic regression analysis was used to identify independent predictors of pain during the procedure. 
Results Male patients were found to be less likely to develop pain during colonoscopy (odds ratio (OR) 0.43 , 95\% CI $0.35-0.53$, $\mathrm{p}<0.001$ ). Those who had any malignancy in the past (OR 0.69 , $95 \%$ CI $0.49-0.97, p=0.02$ ) and previous abdominal surgery (OR $0.63,95 \%$ CI $0.47-0.84, p=0.02$ ) were less prone to having pain. Better bowel preparation improved the comfort of the procedure (OR 0.67, 95\% CI 0.54-0.85, p = 0.001) however those who used Moviprep as bowel preparation were more likely to complain of pain (OR 1.83, 95\% CI 1.34-2.49, p < 0.001). Higher dose of pethidine requirement was found to be associated with increase likelihood of reporting pain (OR 1.03, 95\% CI 1.02-1.04, p < 0.001) but no association was found with the use of midazolam or buscopan. High performance endoscopists were found to cause less pain (OR $0.35,95 \%$ CI $0.27-0.46, p=p<0.001)$. The presence of diverticular disease showed a strong trend towards increasing probability of pain although it did not reach statistical significance (OR1.4, 95\% CI 1.1-1.8, $p=0.07)$. Age and medical co-morbidities like rheumatological and neurological problems did not have any significant association.

Conclusion Likelihood of having abdominal pain during colonoscopy was found to be associated with being female, having poor bowel preparation and the procedure being performed by non-high performing endoscopists. Patients with past history of malignancy were also noted to have less tendency of having pain. The association of higher dose of pethidine and reported pain was likely to reflect the need of larger doses in such situation. Interestingly, history of previous abdominal surgery did not increase the likelihood of reporting pain and in fact had the opposite effect. The reason for why patients who had Moviprep as bowel preparation were more likely to complain of pain is unknown and this may need to be explored in future studies.

Disclosure of Interest None Declared.

\section{PWE-070 THE DUODENAL-JEJUNAL BYPASS SLEEVE (ENDOBARRIER GASTROINTESTINAL LINER) FOR WEIGHT LOSS AND TREATMENT OF TYPE II DIABETES}

doi:10.1136/gutjnl-2013-304907.359

'S Patel, 'D Hakim, ${ }^{3} \mathrm{~J}$ Mason, ${ }^{4,}$ N N Hakim. ' South Thames Foundation School, London, UK; ${ }^{2}$ Royal College of Surgeons in Ireland, Dublin, Ireland; ${ }^{3}$ Gastroenterology, Trafford General Hospital, Manchester; ${ }^{4}$ mmperial College Healthcare NHS Trust, London, UK

Introduction The Duodenal-jejunal bypass sleeve (EndoBarrier Gastrointestinal Liner) is an endoscopically and fluoroscopically inserted implant designed to aid weight loss, treat type II diabetes mellitus and improve the cardiovascular risk profile of subjects. We aimed to trial this device in a cohort of patients to assess efficacy.

Methods We implanted the EndoBarrier bypass sleeve into 57 patients from January 2011 to December 2012. The EndoBarrier is an impermeable fluoropolymer sleeve that is reversibly fixated to the duodenal bulb and extends $80 \mathrm{~cm}$ into the small bowel, usually terminating in the proximal jejunum. It is implanted in the GI tract endoscopically to create a barrier between food and the wall of the intestine and to delay the mixing of digestive enzymes with food. It alters the activation of hormonal signals that originate in the intestine, thus mimicking the effects of a Roux-en-Y gastric bypass procedure without surgery.

Results Results showed weight loss in all patients, as well as lowering of blood sugar levels. Only 1 early device removal (due migration) occurred. There were no major postoperative side effects.

Conclusion Results confirm that the device reduces blood sugar levels and triggers weight loss. This non-permanent device implanted and removed endoscopically, controlled blood sugar and weight loss without the trauma of surgery. Clinical trials to date, involving more than 300 patients, have demonstrated significant weight loss and diabetes improvement with the Endobarrier.
However, since this is a new procedure and due to the lack of data, it is not yet known if weight loss and diabetes benefits will persist. Disclosure of Interest None Declared.

\section{PWE-071 SAFE SEDATION IN ENDOSCOPY: TIME FOR A NEW APPROACH?}

doi:10.1136/gutjnl-2013-304907.360

1."N Zakeri, 'S Webster, 'S Coda, 'A Humphries, ' $\mathrm{A}$ V Thillainayagam. 'Imperial College Healthcare NHS Trust, London, UK

Introduction Despite increasing awareness of the potential hazards of endoscopic sedation, complications from sedation remain a major concern. Serious harm or death resulting from sedation overdose is now a Department of Health 'never event'1. Previous work by our group (2000-2005) demonstrated a steady sedation reversal incidence of $0.27 \%$. Trust guidelines, in line with the current British Society of Gastroenterology safe sedation guidelines $(2003)^{2}$, were developed in 2005 (revised 2011). We present the results of a retrospective survey, evaluating whether the incidence and risk factors for sedation reversal events have changed.

Methods Our Trust is a large tertiary referral endoscopic centre across 3 sites. A retrospective analysis of all endoscopy $(n=73,989)$ was performed, including all sedated endoscopic procedures carried out between 2007 and $2012(\mathrm{n}=52,553)$. Flumazenil or naloxone administration was used as a marker of sedation overdose requiring reversal. The results were compared to the previous single-site audit of 2000 to 2005 ( $n=20,569)$. Reversal episodes were analysed for associations with total sedation dose given, patient ASA grade, age and procedure undertaken. Statistical analysis was carried out using chi squared test and the linear regression model (Origin ${ }^{\circledR}$ ).

Results In total 149 sedation reversals were recorded, representing $0.28 \%$ of all sedated endoscopic procedures, with no significant difference from the reversal rate $(0.27 \%)$ recorded between 2000 and 2005 ( $p=0.79)$. Mean dose of midazolam used in reversal events was $3.1 \mathrm{mg}$ (range $0.5-14 \mathrm{mg}$ ). Mean dose of opioid (as pethidine equivalent) was $47.9 \mathrm{mg}$ (range $12.5-150 \mathrm{mg}$ ). Higher than recommended doses of midazolam $(5 \mathrm{mg})$ or opioid (pethidine equivalent $50 \mathrm{mg}$ ) were administered in $7.4 \%$ and $6.7 \%$ of reversal events, respectively. Endoscopic Retrograde Cholangiopancreatography (ERCP) was most associated with sedation reversal (1\%). Mean dose of midazolam varied by procedure type and was highest for ERCP (5.1 $\pm 2.9 \mathrm{mg})$ and lowest for flexible sigmoidoscopy $(1.7 \pm 0.6 \mathrm{mg}$; $\mathrm{p}<0.01)$. Mean dose of pethidine or opioid equivalent was highest for ERCP $(78 \pm 38.7 \mathrm{mg}$ ) and lowest for colonoscopy $(33 \pm 13.4 \mathrm{mg}$; $p<0.01)$. Sedation reversal was positively associated with increasing patient ASA grade $(\mathrm{p}<0.05)$.

Conclusion Despite the emergence of national and local guidelines, aimed at safe sedation practise, there was no decline in our Trust's rate of sedation reversals over the last 12 years. Furthermore, the findings suggest there is a subgroup of patients, and a subset of endoscopic procedures, which still carry a significant risk of oversedation requiring reversal. This may support the growing interest in alternative sedation strategies for prolonged therapeutic endoscopic procedures such as ERCP.

Disclosure of Interest None Declared.

\section{REFERENCES}

1. DOH 'Never Events' 2012/13

2. Safety and Sedation During Endoscopic Procedures, BSG 2003.

\section{PWE-072 EFFECT OF CHROMOENDOSCOPY ON ADENOMA DETECTION IN THE COLON: A META-ANALYSIS}

doi:10.1136/gutjnl-2013-304907.361

1,2, $\mathrm{N}$ Mohammed, 1,2V Subramanian. ' Gastroenterology, Leeds Teaching Hospitals NHS Trust; ${ }^{2}$ Leeds Institute of Molecular Medicine, Leeds, UK 